\title{
Lifting Techniques: Why Are We Not Using Evidence To Optimize Movement?
}

\author{
Nicholas B. Washmuth, DPT, DMT, OCS ${ }^{1}$, Abby D. McAfee, DPT, OCS ${ }^{2}$, C. Scott Bickel, PhD, PT ${ }^{1}$ \\ 1 Physical Therapy, Samford University, ${ }^{2}$ Saint Louis University; Alton Physical Therapy \\ Keywords: optimize movement, lifting technique, semi-squat lift, squat lift, stoop lift \\ https://doi.org/10.26603/001c.30023
}

\section{International Journal of Sports Physical Therapy}

Vol. 17, Issue 1, 2022

\begin{abstract}
Lifting something off the ground is an essential task and lifting is a documented risk factor for low back pain (LBP). The standard lifting techniques are stoop (lifting with your back), squat (lifting with your legs), and semi-squat (midway between stoop and squat). Most clinicians believe the squat technique is optimal; however, training on squat lifting does not prevent LBP and utilizing greater lumbar flexion (i.e. stoop) when lifting is not a risk factor for LBP. The disconnect between what occurs in clinical practice and what the evidence suggests has resulted in ongoing debate. Clinicians must ask the right questions in order to apply the evidence appropriately. A proposed clinical framework of calm tissue down, build tissue up, improve work capacity can be used to determine which lifting technique is optimal for a patient at any given time. When applying this clinical framework, clinicians should consider metabolic, biomechanical, physical stress tolerance, and pain factors in order to address the movement system. For example, stoop lifting is more metabolically efficient and less challenging to the cardiopulmonary system. There may be few biomechanical differences in spinal postures and gross loads on the lumbar spine between stoop, squat, and semi-squat lifting; however, each lift has distinct kinematic patterns that affects muscle activation patterns, and ultimately the movement system. Clinicians must find the optimal dosage of physical stress to address all aspects of the movement system to minimize the risk of injury. There is no universal consensus on the optimal lifting technique which will satisfy every situation; however, there may be a lifting technique that optimizes movement to achieve a specific outcome. The calm tissue down, build tissue up, improve work capacity framework offers an approach to determine the best lifting technique for an individual patient at any give
\end{abstract} time.

\section{Level of Evidence}

5

\section{PROBLEM}

Lifting something off the ground is an essential task that is required for most individuals to maintain their independence and is also required of athletes for training and performance purposes. Lifting is also a documented risk factor for low back pain (LBP). ${ }^{1}$ Therefore, it is essential that physical therapists provide lifting education and interventions for our patients. The standard lifting techniques: stoop, squat, and semi-squat (Figure 1) are well-described in the literature. ${ }^{2}$ The stoop technique can be quantified as $<45^{\circ}$ knee flexion and $\sim 90^{\circ}$ of trunk flexion, corresponding with a layman's description of lifting with your back. The squat technique can be quantified as $\sim 135^{\circ}$ knee flexion and $<30^{\circ}$ trunk flexion, corresponding with a layman's description of lifting with your legs. The semi-squat technique uses a posture midway between the stoop and squat lifts, which quantitatively can be described as $\sim 90^{\circ}$ knee flexion and $\sim 45^{\circ}$ trunk flexion.

Although $75 \%$ of physiotherapists, $91 \%$ of manual handling advisors, and $88 \%$ of osteopaths believe that the squat technique is the safest way to lift, ${ }^{3,4}$ many recreational and competitive athletes utilize the stoop and semi-squat lifting

\footnotetext{
a Corresponding author:

Nick Washmuth

800 Lakeshore Drive, Birmingham, AL

314-229-8043

nwashmut@samford.edu
} 

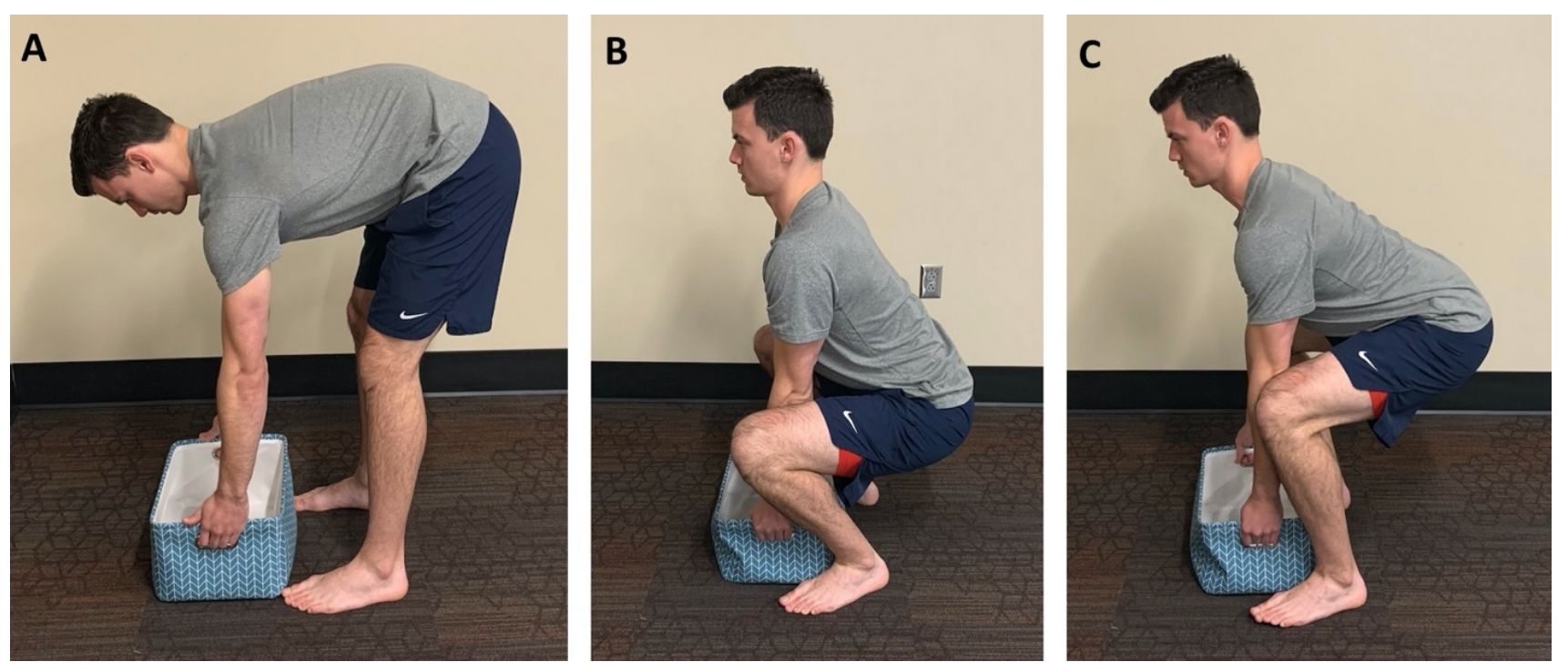

Figure 1. (A) Stoop lift, (B) squat lift, (C) semi-squat lift.

techniques during training. The squat technique is the most commonly advised lifting technique by health care providers $^{5}$, however, it has been shown that training on squat lifting does not prevent $\mathrm{LBP}^{6}$ and utilizing greater lumbar flexion during lifting (i.e. stoop lifting) is not a risk factor for LBP onset, persistence, or recurrence. ${ }^{7}$ There is clearly a disconnect between what occurs in clinical practice and what the evidence suggests related to lifting.

The squat technique is generally accepted to be the optimal lifting technique, ${ }^{2-5}$ but are we really practicing in an evidence-based manner when making this determination? It seems that the answer to the question of "what is the optimal lifting technique" is that "it depends." The authors suggest a more appropriate, alternative question that should be asked: "which lifting technique optimizes movement to complete the task as hand?" The purpose of this clinical suggestion is to propose the calm tissue down, build tissue up, improve work capacity framework that can be used to determine the optimal lifting technique for a patient.

\section{SOLUTION}

The clinical framework of calm tissue down, build tissue up, improve work capacity is the integration of a concept introduced by Greg Lehman, a physiotherapist and chiropractor, and integrated with the the Physical Stress Theory. ${ }^{8}$ One of the primary goals of rehabilitation professionals should be to optimize patients' movement. ${ }^{9}$ Optimizing movement can occur by modulating pain (calm tissue down) to allow for greater options of movement, increasing tissue strength, hypertrophy, neuromuscular activation, power, and/or endurance (build tissue up) to raise the threshold for injury, or increase the total amount of work the body can perform with respect to the muscular and cardiopulmonary systems (improve work capacity). There is an abundance of research and data on lifting, yet clinicians struggle applying the evidence. The calm tissue down, build tissue up, improve work capacity framework can be applied to lifting, in order to utilize evidence appropriately to optimize movement. The
American Physical Therapy Association defines the Movement System as a collection of systems that interact to move the body or its component parts. ${ }^{9}$ Since human movement is a complex behavior within a specific context, ${ }^{9}$ the authors consider four primary movement factors when applying this framework: 1) metabolic, 2) biomechanical, 3) physical stress tolerance, and 4) pain.

\section{METABOLIC}

Energy consumption, ventilation, and heart rate are all higher during squat and semi-squat lifting when compared to stoop lifting. ${ }^{2,10}$ In other words, the stoop technique is more metabolically efficient and energy sparing, which is likely the reason many individuals use the stoop as their default lifting technique. ${ }^{2}$ If a goal is to improve work capacity, the patient's intended type of work must be considered. Does the patient require energy conservation for repetitive submaximal lifting and work productivity? If so, the stoop technique would be a more optimal movement as it is more energy efficient, less challenging to the cardiopulmonary system, and has been shown to be a quicker technique. ${ }^{2}$

\section{BIOMECHANICAL}

During lifting, the lumbar spine is subjected to high loads; however, it is not clear if loads differ between lifting techniques. $^{2,11}$ It appears that joint positions, the size and weight of the load lifted, and the biomechanical models used to estimate forces affect the calculated loads on the spine. ${ }^{2,11}$ It has also been shown that the lumbar spine experiences significant amounts of flexion, even when subjects attempt to keep a "neutral" spine, ${ }^{12}$ suggesting there may be very little difference in spinal postures between the stoop and squat techniques. Even with the unclear difference in the gross loads placed on the lumbar spine, ${ }^{2,11}$ as indicated by estimated moments acting on the spine, and unclear differences in spinal postures between the lifting techniques, evidence suggests subjects with LBP, when 
asked to lift, utlize the squat technique, ${ }^{13}$ indicating the squat techqnique may feel less stressful. Looking collectively at the overall loads on the spine and the relative load sharing between active and passive spinal structures, there is evidence to support the utilization of semi-squat lifting. ${ }^{14}$

It is clear that the kinematic patterns for the stoop, squat, and semi-squat techniques are different. The most obvious difference is that stoop lifting requires greatest trunk flexion, the squat lift requires greater tibiofemoral flexion, and the semi-squat lift is a blend of squat and stoop lifts. ${ }^{15}$ These kinematic differences will affect muscle activation patterns, power generation abilities, and joint stress. ${ }^{15}$ If the goal is to optimize movement by building tissue up, the therapist needs to consider prescribing the lifting technique that produces the desired muscle activity and power generation in the targeted tissue(s). The squat technique is optimal for quadriceps muscle activation, while the stoop technique is optimal for hamstring and lumbar extensor muscle activation. ${ }^{15,16}$ If the goal is to optimize movement by calming tissue down, the therapist should prescribe the lifting technique that incorporates kinematic patterns that do not excessively load tissues that may not be able to currently handle the stress. Based on what is known on the effects of different kinematic patterns on lifting, athletes and fitness populations need to ensure they utilize a lifting technique that accomplishes their desired outcome. Specific kinematic patterns used during a lift can help to protect painful tissue, help to strengthen specific tissue, and can ultimately help create an advantage when lifting heavy loads.

The amount of weight someone is able to lift is influenced by their lifting technique. There is strong evidence suggesting individuals self-select a maximum acceptable weight for the semi-squat and stoop lifts that is greater than the squat lift; however, the strength capacity of each lifting technique is very similar. ${ }^{2}$ A recent study looked at the effect of three different lumbar postures while lifting with the knees in $45^{\circ}$ of flexion on lumbar extensor strength, which is a technique that would fall somewhere between the stoop and semi-squat lifting techniques. ${ }^{16}$ The three different lumbar postures included full extension (lordotic), mid-range (flat back), and fully flexed. It was found that lumbar extensor moments and neuromuscular efficiency was greatest when lifting with fully flexed lumbar spines and changes in lumbar spine posture did not influence hip or knee moments. ${ }^{16}$ These findings suggest that lifting with your legs while keeping your back straight (squat lifting) may not be an efficient way to lift heavy loads. ${ }^{16}$

\section{PHYSICAL STRESS TOLERANCE}

Changes in the relative level of physical stress on the body will cause predictable adaptive responses in biological tissue. ${ }^{8}$ Tissue will atrophy under decreased stress, be maintained when stress is unchanged, and will hypertrophy under increased stress. ${ }^{8}$ Increased stress will also provide an opportunity for development of increased strength, neuromuscular activation, power, and/or endurance. Tissue will become injured if exposed to stress beyond its physical capacity. ${ }^{8}$
There is a common belief among clinicians that stoop technique leads to intervertebral disc herniations, which is a primary reason clinicians oppose stoop lifting. While herniations are associated with spinal compressive loads in flexion (i.e. stoop lifting), there is a high percentage of asymptomatic individuals with herniations ${ }^{16}$ and spontaneous regression of herniated disc tissue can occur. ${ }^{17}$ In line with other tissues, such as bone and muscle, specific types of loading appear to be beneficial to the intervertebral disc and will result in hypertrophy ${ }^{18}$ (build tissue up) or spontenous regression of disc material. ${ }^{19}$ Intervertebral discs act as shock-absorbing cushions between vertebrae, and, if compressed or degenerative, may lose flexibility and load bearing ability. It is unknown if intervertebral disc hypertrophy is clinically revelant or protective; however, hypertrophy appears to be possible in the intervertebral disc. ${ }^{18}$ It is currently unknown what mode of stress or dosage is optimal for intervertebral disc hypertrophy. If a clinician's goal is to optimize movement by building tissue up, then physical stress levels that overload the tissue are required. However, excessively high levels of physical stress may result in injury. Therefore, it is the clinician's job to find the optimal dosage of physical stress to ensure hypertrophy and minimize the risk of injury, and impact the movement system, even in those with intervertebral disc pathologies.

When considering modification of a patient's lifting technique, clinicians must consider whether it was the technique itself or the inadequate dosage of physical stress that led to injury. Changing the patient's preferred lifting technique may change the physical stress patterns on their body, decreasing stress in some areas while increasing stress to other areas which could result in pain in other areas.

\section{PAIN}

Pain is a perception and one of the ways the brain lets us know that it perceives a threat. If a patient has pain with lifting, should the clinician permanently change the way the patient lifts or utilize an alternative lifting technique as an intervention until symptoms resolve? If a patient is in pain, sometimes the goal is to give that perceived threat a break (calm tissue down). Recognizing that pain is complex and interrelated with many other variables, the challenge clinicians have is determining when they should be calming tissue down, building tissue up, or improving work capacity to optimize movement. With an acute injury and intense pain, the clinical focus should be to protect the tissue by decreasing the physical stress, as the tissue is likely not prepared to handle load and is at risk for further injury. This can be accomplished by educating a patient on alternate lifting techniques that can be utilized in the presence of pain. An alternate lifting technique as a temporary desensitizer can be used to calm tissue down prior to building tissue up. Even in cases of chronic pain, alternate lifting techniques can be used to teach the patient that they can control their pain and positively affect overall movement tolerance. 


\section{Clinical Formula: Calm Tissue Down $\longrightarrow$ Build Tissue Up $\longrightarrow$ Improve Work Capacity}

\begin{tabular}{|c|c|c|c|}
\hline \multirow{2}{*}{ Factors to Consider: } & $\begin{array}{l}\text { Pain: } \\
\text { Decreased Perceived Threat } \\
\text { Control Pain }\end{array}$ & $\begin{array}{l}\text { Biomechanical: } \\
\text { Increase Tissue Strength } \\
\text { Muscle Activation }\end{array}$ & $\begin{array}{l}\text { Metabolic: } \\
\text { Energy Sparing } \\
\text { Biomechanical: }\end{array}$ \\
\hline & $\frac{\text { Physical Stress Tolerance: }}{\text { Decrease Stress }}$ & $\begin{array}{l}\text { Physical Stress Tolerance: } \\
\text { Optimal Dosage for Hypertrophy }\end{array}$ & $\begin{array}{l}\text { Biomechanical: } \\
\text { Force Developmen }\end{array}$ \\
\hline & & $\downarrow$ & $\downarrow$ \\
\hline Lifting Technique: & \multicolumn{3}{|c|}{ Squat, Stoop, or Semi-Squat: Can a single lifting technique accomplish it all? } \\
\hline
\end{tabular}

Figure 2. Application of Calm Tissue Down, Build Tissue Up, Improve Work Capacity framework to determine appropriate lifting technique.

\section{DISCUSSION}

The conflicting evidence regarding lifting techniques implies that there is no universal consensus on the optimal lifting technique; however, there may be a lifting technique that optimizes movement to complete the task at hand. A lifting technique should be specific to a patient's desired outcome and tailored to improve their unique function (Figure 2). Clinicians need to consider why they would promote one lifting technique over another, at any given time. The following clinical examples highlight the application of this clinical framework.

In CrossFit, the athlete that completes a workout in the shortest time "wins" that workout. Depending on the specifics of a CrossFit workout, the athlete will require varying degrees of strength, neuromuscular activation, power, and endurance. Similar to a track athlete completing a mile run as quickly as possible, CrossFit athletes complete many workouts as fast as they can. An example of a CrossFit workout might be completing 21 deadlift repetitions, 21 handstand push-up repetitions, 15 deadlift repetitions, 15 handstand push-up repetitions, 9 deadlift repetitions, and 9 handstand push-up repetitions, as fast as possible. The deadlift exercise requires lifting a barbell off the ground until the athlete is standing erect. The goal is to complete the deadlifts as quickly as possible, while also conserving as much energy as possible, in order to complete the handstand push-ups quickly, which would ultimately lead to a faster time and improve the athlete's chances of winning. A lifting technique that resembles the stoop technique may be most optimal for this athlete, as the stoop lift is more energy efficient, less challenging to the cardiopulmonary system, and has been shown to be a quicker technique. ${ }^{2}$ Lumbar extensor moments and neuromuscular efficiency when deadlifting is greater when the lumbar spine is flexed, ${ }^{16}$ providing another potential benefit of stoop lifting for this athlete.
Patellofemoral pain has an annual prevalence in the general population of over $22 \%$ and over $35 \%$ in professional male cyclists. ${ }^{20}$ The incidence rates for patellofemoral pain in adolescent amateur athletes is between $5.1 \%-14.9 \%{ }^{20}$ Due to the high incidence and prevalence rates for for patellofemoral pain and poor long term prognosis, ${ }^{20}$ optimizing treatment in these patients should be a priority. Patellofemoral joint reaction forces are partially explained by the knee flexion angle; as knee flexion increases in a closed chain environment, the patellofemoral compressive load is increased. ${ }^{21}$ However, quadriceps strengthening exercises should be included in the management of patients with patellofemoral pain. ${ }^{22}$ Those patients with high levels of patellofemoral pain may benefit from stoop lifting, where the knee flexion angle is minimal and remains relatively static, while those patients with resolving or lower pain may benefit from squat lifting for optimal quadriceps activation. ${ }^{15}$

A patient presents with a discogenic LBP, where their pain is reproduced with prolonged sitting, forward bending, and lifting anything heavier than 10 pounds. In an acute case, this patient would benefit from a lifting technique that prevents exacerbation of symptoms (calm tissue down), and in a chronic case, a lifting technique should be used that allows the patient to control or manage their symptoms while maintaining their independence (improving work capacity). Evidence suggests that patients with LBP prefer the squat technique, ${ }^{13}$ indicating that squat lifting may be best indicated for this patient while in the acute stage.

Evidence supports each technique in different scenarios; therefore, clinicians need to start asking alternative questions: How can movement be optimized by calming tissue down, building tissue up, or improving work capacity? This question cannot be answered without in depth, integrative knowledge of the movement system and its component elements. ${ }^{9}$ 
FINANCIAL DISCLOSURE AND CONFLICT OF INTEREST

None

\section{ACKNOWLEDGEMENTS}

The authors would like to thank Samford University's Doctor of Physical Therapy class of 2023 for engaging in a lively class debate which served as the genesis for this manuscript.

Submitted: February 22, 2021 CST, Accepted: August 23, 2021 CST 


\section{REFERENCES}

1. Goncharenko IM, Komleva NE, Chekhonatsky AA. Lower back pain at workplace: Prevalence and risk factors. Russ Open Med J. 2020;9(2). doi:10.15275/rus omj.2020.0207

2. Straker LM. A review of research on technique for lifting low-lying objects: 2 . Evidence for a correct technique. Work. 2003;20(2):83-96.

3. Nolan D, O’Sullivan K, Stephenson J, O’Sullivan P, Lucock M. What do physiotherapists and manual handling advisors consider the safest lifting posture, and do back beliefs influence their choice?

Musculoskelet Sci Pract. 2018;33:35-40. doi:10.1016/ j.msksp.2017.10.010

4. Smith K, Thomson OP. What do UK osteopaths view as the safest lifting posture, and how are these views influenced by their back pain beliefs? Int J Osteopath Med. 2020;37:10-16. doi:10.1016/j.ijosm.20 $\underline{20.07 .003}$

5. Abdoli-Eramaki M, Agababova M, Janabi J, Pasko E, Damecour C. Evaluation and comparison of lift styles for an ideal lift among individuals with different levels of training. Appl Ergon. 2019;78:120-126. doi:1 0.1016/j.apergo.2019.02.007

6. Denis D, Gonella M, Comeau M, Lauzier M. Questioning the value of manual material handling training: A scoping and critical literature review. Appl Ergon. 2020;89. doi:10.1016/j.apergo.2020.103186

7. Saraceni N, Kent P, Ng L, Campbell A, Straker L, O'Sullivan P. To Flex or Not to Flex? Is There a relationship between lumbar spine flexion during lifting and low back pain? A systematic review with meta-analysis. J Orthop Sports Phys Ther. 2020;50(3):121-130. doi:10.2519/jospt.2020.9218

8. Mueller MJ, Maluf KS. Tissue adaptation to physical stress: A proposed "physical stress theory" to guide physical therapist practice, education, and research. Phys Ther. 2002;82(4):382-403. doi:10.1093/ptj/82.4.3 $\underline{83}$

9. American Physical Therapy Association. Physical Therapist Practice and the Movement System [White Paper].; 2015.

10. Wang Z, Wu L, Sun J, He L, Wang S, Yang L. Squat, stoop, or semi-squat: A comparative experiment on lifting technique. J Huazhong Univ Sci Technol [Med Sci]. 2012;32(4):630-636. doi:10.1007/s11596-012-100 9-3
11. Dreischarf M, Rohlmann A, Graichen F, Bergmann $\mathrm{G}$, Schmidt $\mathrm{H}$. In vivo loads on a vertebral body replacement during lifting techniques. J Biomech. 2016;49(6):890-895. doi:10.1016/j.jbiomech.2015.0 $\underline{9.034}$

12. Aasa U, Bengtsson V, Berglund L, Öhberg F. Variability of lumbar spinal alignment among powerand weightlifters during the deadlift and barbell back squat. Sports Biomech. Published online 2019:1-17. do i:10.1080/14763141.2019.1675751

13. Nolan D, O'Sullivan K, Newton C, Singh G, Smith $\mathrm{BE}$. Are there differences in lifting technique between those with and without low back pain? A systematic review. Scand J Pain. 2020;20(2):215-227. doi:10.1515/ sjpain-2019-0089

14. Khoddam-Khorasani P, Arjmand N, Shirazi-Adl A. Effect of changes in the lumbar posture in lifting on trunk muscle and spinal loads: A combined in vivo, musculoskeletal, and finite element model study. $J$ Biomech. 2020;104. doi:10.1016/j.jbiomech.2020.1097 $\underline{28}$

15. van der Have A, Van Rossom S, Jonkers I. Squat lifting imposes higher peak joint and muscle loading compared to stoop lifting. Appl Sci. 2019;9(18):3794. doi:10.3390/app9183794

16. Mawston G, Holder L, O’Sullivan P, Boocock M. Flexed lumbar spine postures are associated with greater strength and efficiency than lordotic postures during a maximal lift in pain-free individuals. Gait \& Posture. 2021;86:245-250. doi:10.1016/j.gaitpost.202 $\underline{1.02 .029}$

17. Brinjikji W, Luetmer PH, Comstock B, et al. Systematic literature review of imaging features of spinal degeneration in asymptomatic populations. Am J Neuroradiol. 2015;36(4):811-816. doi:10.3174/aj nr.a4173

18. Belavý DL, Quittner MJ, Ridgers N, Ling Y, Connell $\mathrm{D}$, Rantalainen T. Running exercise strengthens the intervertebral disc. Sci Rep. 2017;7(1). doi:10.1038/sre p45975

19. Chiu CC, Chuang TY, Chang KH, Wu CH, Lin PW, Hsu WY. The probability of spontaneous regression of lumbar herniated disc: A systematic review. Clin Rehabil. 2015;29(2):184-195. doi:10.1177/0269215514 $\underline{540919}$ 
20. Smith BE, Selfe J, Thacker D, et al. Incidence and prevalence of patellofemoral pain: A systematic review and meta-analysis. PLOS ONE.

2018;13(1):e0190892. doi:10.1371/journal.pone.0190 $\underline{892}$

21. Levangie PK, Norkin CC, Lewek MD. Joint Structure \& Function. A Comprehensive Analysis. 6th ed. FA Davis; 2019.
22. Willy RW, Hoglund LT, Barton CJ, et al. Patellofemoral pain. J Orthop Sports Phys Ther. 2019;49(9):CPG1-CPG95. doi:10.2519/jospt.2019.030 2 